\title{
Rapport annuel du Système canadien de surveillance des effets secondaires suivant l'immunisation (SCSESSI) pour les vaccins administrés en 2012
}

\author{
Law $B J^{1^{*}}$, Laflèche $\mathrm{J}^{1,2}$, Ahmadipour $\mathrm{N}^{1}$, Anyoti $\mathrm{H}^{1}$ \\ ${ }^{1}$ Centre de l'immunisation et des maladies respiratoires infectieuses, Agence de la santé publique du Canada, Ottawa \\ (Ontario) \\ ${ }^{2}$ Citoyenneté et Immigration Canada, Ottawa (Ontario) \\ ${ }^{*}$ Correspondance : Barbara.law@phac-aspc.gc.ca
}

\section{Résumé}

Contexte : Décrire le profil de déclaration des effets secondaires suivant l'immunisation (ESSI) pour les vaccins administrés au Canada en 2012 ainsi que les tendances d'évolution des taux de déclaration d'effets secondaires pour les vaccins administrés de 2005 jusqu'à la fin de 2011.

Méthodologie : Analyse de données fondée sur les déclarations d'ESSI reçues par l'Agence de la santé publique du Canada en date du 30 avril 2013, pour les vaccins commercialisés au Canada et administrés entre le $1^{\text {er }}$ janvier 2005 et le 31 décembre 2012.

Résultats : Le taux de déclaration des ESSI était de 10,1 pour 100000 habitants au Canada pour les vaccins administrés en 2012; il était inversement proportionnel à l'âge des personnes vaccinées. On observe une tendance à la baisse des taux de $2005(14,8)$ à 2012 sur l'ensemble de la population et en fonction de l'âge. Dans la grande majorité des cas, les effets déclarés (94 à $95 \%$ ) n'étaient pas graves et consistaient en des réactions au point de vaccination ou à proximité, une éruption cutanée et de la fièvre.

Conclusion : Le Canada bénéficie d'un robuste système de pharmacovigilance vaccinale et jouit de l'un des taux de déclaration d'ESSI les plus élevés des pays développés. Les vaccins commercialisés au Canada possèdent un très bon profil d'innocuité. Ce rapport permet d'établir des comparaisons à l'échelle des provinces et territoires du Canada et à l'échelle mondiale.

\section{Introduction}

Le Système canadien de surveillance des effets secondaires suivant l'immunisation (SCSESSI) est un effort collaboratif des autorités de santé publique fédérales, provinciales et territoriales qui date de 1987 (1, 2). À l'échelon national, le SCSESSI relève de la Section de la sécurité des vaccins du Centre de l'immunisation et des maladies respiratoires infectieuses de l'Agence de la santé publique du Canada (ASPC). Des rapports nationaux sur la sécurité des vaccins sont publiés régulièrement $(2,3,4,5,6,7,8,9,10,11)$. Le dernier rapport publié mettait l'accent sur les données de surveillance de la sécurité des vaccins administrés en 2004 et sur les tendances annuelles de déclaration remontant jusqu'en 1992 (11). Le présent rapport a pour but principal de résumer les déclarations d'effets secondaires suivant l'immunisation (ESSI) reçues par l'ASPC pour les vaccins administrés en 2012 et d'offrir des données comparatives annuelles pour les vaccins administrés entre 2005 et la fin de 2011. II inaugure également le format normalisé adopté pour les futurs rapports annuels qui facilitera la comparaison des données de déclaration d'année en année ainsi que la comparaison avec les rapports fournis par d'autres provinces et territoires du Canada (12) et d'autres pays (13-14). 
Les objectifs du SCSESSI sont les suivants : assurer une surveillance continue de la sécurité des vaccins commercialisés au Canada; détecter l'augmentation de la fréquence ou de la gravité des réactions liées aux vaccins recensées par le passé; détecter des ESSI jusque-là inconnus susceptibles d'être liés à un vaccin; cerner les domaines nécessitant la réalisation d'investigations ou de recherches plus poussées; fournir une information opportune sur les profils de déclaration des ESSI pour les vaccins commercialisés au Canada, apte à favoriser une prise de décisions éclairée en matière d'immunisation (15).

Afin de réaliser ces objectifs, le SCSESSI s'appuie sur une surveillance passive améliorée ainsi que sur une surveillance syndromique active. La première comprend tous les systèmes en place pour surveiller les cas d'ESSI et les déclarer aux programmes d'immunisation provinciaux, territoriaux et fédéraux du Canada; elle est dite " améliorée " car elle a toujours mis au premier plan la déclaration des effets secondaires reconnus comme particulièrement importants pour la santé publique, lesquels sont présentés accompagnés d'une case à cocher sur le formulaire national de déclaration des ESSI (16). L'ensemble des provinces et des territoires encouragent activement la déclaration des ESSI dans leurs régions (17).

La surveillance syndromique active est menée depuis 1991 dans le cadre du Programme de surveillance active de l'immunisation (IMPACT) (18). Le Programme IMPACT est financé par l'Agence de la santé publique du Canada en vertu d'un contrat passé avec la Société canadienne de pédiatrie et comprend douze centres pédiatriques à l'échelle du Canada, qui représentent plus de $90 \%$ de toutes les admissions en soins tertiaires pédiatriques au pays $(19,20)$. Le programme IMPACT examine les admissions hospitalières pour cause d'événements neurologiques (p. ex. convulsions, encéphalite, paralyse flasque aiguë, notamment syndrome de Guillain-Barré, méningite à liquide clair), de thrombocytopénie, d'abcès/cellulite au point de vaccination et d'autres complications potentiellement consécutives à l'administration de vaccins. Tout événement jugé comme étant lié de façon temporaire à l'administration d'un vaccin, pour lequel aucune explication claire n'est donnée, est déclaré en tant qu'ESSI.

Les identifiants personnels sont supprimés de toutes les déclarations d'ESSI émanant des autorités fédérales, provinciales et territoriales et du programme IMPACT avant que celles-ci ne soient transmises à l'ASPC pour être regroupées dans une base de données nationale à des fins de détection de signes et de production de rapports. À partir de 1987 et jusqu'à la fin de 2010, les détenteurs d'une autorisation de mise en marché déclaraient également les ESSI à I'ASPC. En janvier 2011, la réglementation en matière de déclarations des ESSI a été remaniée et les détenteurs d'une autorisation de mise en marché sont désormais tenus de transmettre leurs déclarations directement à Santé Canada; depuis, plusieurs d'entre eux ont cessé d'adresser leurs déclarations à I'ASPC.

Vous trouverez une explication plus détaillée des rôles et responsabilités respectifs en matière de pharmacovigilance postcommercialisation qui incombent à Santé Canada, à l'ASPC et aux autorités d'immunisation fédérales, provinciales et territoriales, à la section intitulée « Innocuité des vaccins » du Guide canadien d'immunisation (21) et sur la page du site Web du SCSESSI (15).

\section{Méthodologie}

\section{Traitement des déclarations reçues par le SCSESSI}

Toutes les déclarations d'ESSI sont entrées dans la base de données du SCSESSI et codées conformément à la terminologie de MedDRA (Dictionnaire médical international des activités de réglementation). Un examen systématique des dossiers médicaux est également effectué par des professionnels de la santé qualifiés afin de classer chaque déclaration en fonction de la raison unique la plus importante à son origine (principal ESSI, (tableau 1) et de la gravité des effets déclarés (tableau 2). 
Tableau 1 : Catégories et sous-types d'événements secondaires suivant l'immunisation (ESSI) utilisés dans l'examen des dossiers médicaux à des fins de classification

\begin{tabular}{|c|c|}
\hline Catégorie & Sous-types de catégories d'ESSI \\
\hline $\begin{array}{l}\text { Réactions au site de vaccination ou } \\
\text { à proximité }\end{array}$ & $\begin{array}{l}\text { Abcès (infecté ou stérile) } \\
\text { Cellulite } \\
\text { OEdème sévère d'un membre } \\
\text { Douleur au membre vacciné durant } 7 \text { jours ou plus } \\
\text { Autre réaction locale }\end{array}$ \\
\hline $\begin{array}{l}\text { Événements allergiques ou de type } \\
\text { allergique }\end{array}$ & $\begin{array}{l}\text { Anaphylaxie } \\
\text { Syndrome oculo-respiratoire (SOR) } \\
\text { Autres événements allergiques }\end{array}$ \\
\hline Événements neurologiques & $\begin{array}{l}\text { Encéphalite/encéphalomyélite aiguë disséminée/myélite } \\
\text { Ataxie cérébelleuse } \\
\text { Méningite à liquide clair } \\
\text { Syndrome de Guillain-Barré (SGB) } \\
\text { Paralysie de Bell } \\
\text { Autre paralysie persistant au-delà de } 1 \text { jour } \\
\text { Convulsions } \\
\text { Autre manifestation neurologique }\end{array}$ \\
\hline Éruption cutanée seulement & Généralisée, localisée ou site non précisé \\
\hline $\begin{array}{l}\text { Autres événements indiqués sur le } \\
\text { formulaire de déclaration du } \\
\text { SCSESSI }\end{array}$ & $\begin{array}{l}\text { Arthrite } \\
\text { Épisode hypotonique-hyporéactif }(\mathrm{EHH}) \\
\text { Intussusception } \\
\text { Paresthésie/anesthésie } \\
\text { Parotidite } \\
\text { Pleurs persistants } \\
\text { Thrombocytopénie }\end{array}$ \\
\hline $\begin{array}{l}\text { Autres effets généraux non } \\
\text { précisés sur le formulaire de } \\
\text { déclaration du SCSESSI }\end{array}$ & $\begin{array}{l}\text { Fièvre seule, infection, syndrome grippal, autre(s) symptôme(s) généraux, éruption } \\
\text { cutanée avec fièvre ou autre syndrome médical }\end{array}$ \\
\hline $\begin{array}{l}\text { Effets liés à l'anxiété lors de la } \\
\text { vaccination }\end{array}$ & (Aucun sous-type) \\
\hline Autres événements divers & $\begin{array}{l}\text { Troubles gastro-intestinaux } \\
\text { Échec du vaccin } \\
\text { Autres événements }\end{array}$ \\
\hline $\begin{array}{l}\text { Erreur de vaccination sans ESSI } \\
\text { associé }\end{array}$ & (Aucun sous-type) \\
\hline
\end{tabular}

Tableau 2 : Critères de classification des effets secondaires suivant l'immunisation (ESSI) en fonction de leur degré de gravité utilisés dans l'examen des dossiers médicaux

\begin{tabular}{|c|c|}
\hline Gravité & Critère \\
\hline Graves & $\begin{array}{ll}\text { - } & \text { Issue fatale } \\
\text { - } & \text { Entraîne l'hospitalisation ou le prolongement de l'hospitalisation du patient pour une durée d'au } \\
\text { moins } 24 \text { heures } \\
\text { - } \quad \text { Entraîne une invalidité ou une incapacité persistante ou marquée } \\
\text { - } \quad \text { Entraîne une anomalie ou une déficience congénitale } \\
\text { Menace vitale }\end{array}$ \\
\hline $\begin{array}{l}\text { Répercussions } \\
\text { élevées }\end{array}$ & $\begin{array}{l}\text { - } \quad \text { Entraîne une hospitalisation de moins de } 24 \text { heures } \\
\text { - } \quad \text { Exige une observation extra-hospitalière sous surveillance médicale } \\
\text { - } \quad \text { Exige au moins } 3 \text { évaluations médicales séparées lors de la survenue d'un épisode aigu d'ESSI } \\
\text { - Exige l'administration d'un traitement par voie intraveineuse en ambulatoire (p. ex. antibiothérapie } \\
\text { ou réhydratation) }\end{array}$ \\
\hline
\end{tabular}




\begin{tabular}{|l|ll|}
\hline & $\bullet$ & Empêche la réalisation des activités quotidiennes pendant au moins 4 jours \\
\hline Répercussions & $\bullet$ & Entraîne 1 à 2 évaluations médicales non prévues, urgentes ou non urgentes \\
modérées & $\bullet$ & Exige la venue des services médicaux d'urgence à la clinique de vaccination \\
& - & Entraîne la prescription d'un nouveau médicament ou l'augmentation de la dose d'un médicament \\
& actuel & Empêche la réalisation des activités quotidiennes pendant 1 à 3 jours \\
\hline Répercussions faibles & - & Exige l'administration d'un traitement au sein de la clinique de vaccination uniquement, prodigué \\
& & par le personnel de la clinique \\
& - & Exige les conseils et la rassurance d'un professionnel de la santé sans prise de rendez-vous \\
& - & Exige la prise d'un médicament en vente libre pour le soulagement des symptômes \\
& - & Empêche la réalisation des activités quotidiennes pendant moins de 24 heures \\
& - & Aucune répercussion discernable \\
\hline
\end{tabular}

\section{Principales définitions}

Les effets secondaires suivant l'immunisation (ESSI) désignent généralement tout événement médical fâcheux qui se produit à la suite de l'administration d'un vaccin et qui n'a pas nécessairement de lien causal avec celle-ci. Un effet secondaire peut donc correspondre à un signe défavorable ou non intentionnel, à un résultat de laboratoire anormal, à un symptôme ou à une affection (22).

En 1994, la Conférence internationale d'harmonisation sur les exigences techniques relatives à l'homologation des produits pharmaceutiques à usage humain a établi des critères de gravité pour favoriser une déclaration rapide des ESSI (23). Un ESSI grave (EIG) est un ESSI qui entraîne la mort, menace le pronostic vital, exige l'hospitalisation du patient ou la prolongation de son hospitalisation, entraîne une invalidité ou une incapacité persistante ou marquée, ou une déficience ou une malformation congénitale (23). On entend par déclaration rapide, une déclaration effectuée dans un délai maximal de 15 jours après la détection de l'ESSI ou plus tôt si possible, de préférence. À l'ASPC, toutes les déclarations d'EIG sont examinées par un médecin.

Un ESSI inattendu est un effet indésirable dont la nature ou la gravité ne correspondent pas aux indications portées sur l'étiquette canadienne du produit ni aux caractéristiques attendues du vaccin (23).

La classification des ESSI effectuée dans le cadre de l'examen des dossiers médicaux s'est appuyée sur les définitions nationales de cas, le cas échéant, notamment les définitions publiées de Brighton Collaboration $(24,25,26,27,28,29,30,31,32,33,34,35,36,37)$ ainsi que les définitions adoptées dans le Guide de l'utilisateur du SCSESSI pour le syndrome oculo-respiratoire, la paralysie de Bell, l'arthrite, la parotidite, l'anesthésie et la paresthésie (38).

Autres définitions utilisées :

- Gonflement étendu d'un membre qui touche toute la longueur d'un segment proximal ou distal du membre, le segment étant la partie d'un membre comprise entre deux articulations (39).

- Ataxie cérébelleuse : apparition soudaine d'une ataxie du tronc et de troubles de la démarche (40). À noter que cela suppose l'absence de tout signe cérébelleux associé à d'autres symptômes d'encéphalite ou d'encéphalomyélite disséminée aiguë, auquel cas la définition de cas de Brighton serait utilisée (28).

\section{Collecte et analyse des données}

Toutes les déclarations d'ESSI reçues en date du 30 avril 2013, pour lesquelles la date d'administration des vaccins se situait entre le $1^{\mathrm{er}}$ janvier 2005 et le 31 décembre 2012, ont été extraites de la base de données du SCSESSI. Les déclarations concernant uniquement l'administration de l'un des vaccins contre la grippe pandémique H1N1 ont été exclues car ces produits ont été utilisés exclusivement en 2009 et 2010, ce qui aurait introduit un facteur de confusion dans la comparaison entre les tendances de déclaration observées pour les vaccins administrés en 2012 et celles concernant les vaccins administrés de 2005 à 2011. 
Toutes les analyses de données ont été menées à l'aide du logiciel d'analyse statistique SAS 9.3. Les taux de déclaration pour 100000 habitants ont été calculés à partir des estimations annuelles de la population par âge, sexe, province et territoire, couvrant la période de 2005 à 2012 (41).

Les renseignements relatifs au total des doses de vaccin distribuées en 2011 et 2012 ont été fournis par les détenteurs d'autorisation de mise en marché et sont considérés comme étant de nature exclusive. Afin de permettre de présenter la totalité des déclarations d'ESSI et d'EIG pour les vaccins administrés en 2012 et les taux de déclaration pour 100000 doses distribuées sans qu'il soit possible de calculer le total annuel des doses distribuées, les taux de déclaration ont été calculés à partir des données regroupées de 2011 et de 2012. Les vaccins ont été groupés en fonction de leur contenu antigénique aux fins de l'analyse.

\section{Résultats}

\section{Nombre total d'effets et taux de déclaration}

Sur les 38364 déclarations d'ESSI extraites, 5204 concernant uniquement le vaccin contre la grippe pandémique ont été exclues, ce vaccin n'ayant été utilisé qu'en 2009 et 2010. Sur les 33160 déclarations incluses dans l'analyse, les déclarations d'ESSI (\% EIG), par année d'administration des vaccins, étaient réparties comme suit : 2005 : 4792 (4,5\%); 2006 : 4417 (4,8\%); 2007 : 4258 (5,3\%); 2008 : 4482 (4,7\%); 2009 : 4099 (5,8 \%); $2010: 4046$ (5,9\%); $2011: 3558$ (5,8\%); $2012: 3508$ (5,4\%).

Le taux de déclaration en fonction de l'âge pour 100000 habitants par année d'administration des vaccins pour l'ensemble du Canada apparaît au tableau 3. Sur l'ensemble de la période d'administration des vaccins évaluée, les taux de déclaration les plus élevés ont été observés chez les enfants âgés entre 1 et 2 ans, suivis des nourrissons de moins de 1 an. Une forte baisse est observée chez les enfants âgés entre 2 et 7 ans, ceux âgés entre 7 et 18 ans et chez les adultes à partir de 18 ans. On observe également une tendance régulière à la baisse dans les taux de déclaration tout au long de la période étudiée, plus particulièrement chez les enfants de moins de 7 ans et pour les ESSI plus que pour les EIG.

Tableau 3 : Taux de déclaration annuel des $\mathrm{ESSI}^{1}$ et des $\mathrm{EIG}^{2}$ en fonction de l'âge pour 100000 habitants pour les vaccins administrés de 2005 jusqu'à la fin de 2012

\begin{tabular}{|c|c|c|c|c|c|c|c|c|}
\hline \multirow{2}{*}{ Groupe d'âge } & \multicolumn{8}{|c|}{ Taux de déclaration des ESSI (EIG) pour 100000 habitants } \\
\hline & 2005 & 2006 & 2007 & 2008 & 2009 & 2010 & 2011 & 2012 \\
\hline$<1$ an & $176(16)$ & $161(16)$ & $169(18)$ & $134(12)$ & 152(19) & $150(20)$ & $136(15)$ & $130(12)$ \\
\hline de 1 à $<2$ ans & $305(22)$ & $290(24)$ & $276(22)$ & 283(22) & 238(18) & $217(18)$ & 202(17) & 152(16) \\
\hline de 2 à $<7$ ans & $47,1(1,2)$ & $36,7(1,1)$ & $31,5(1,2)$ & $31,0(1,2)$ & $27,8(1,0)$ & $28,7(1,0)$ & $28,8(1,4)$ & $25,2(1,2)$ \\
\hline de 7 à $<18$ ans & $11,9(0,5)$ & $11,4(0,4)$ & $9,5(0,4)$ & $15,1(0,6)$ & $12,3(0,5)$ & $12,0(0,4)$ & $9,7(0,6)$ & $11,2(0,4)$ \\
\hline de 18 à $<65$ ans & $6,5(0,2)$ & $6,0(0,1)$ & $6,0(0,1)$ & $5,6(0,1)$ & $4,9(0,2)$ & $4,7(0,1)$ & $4,2(0,1)$ & $5,0(0,1)$ \\
\hline 65 ans et plus & $8,0(0,3)$ & $6,6(0,2)$ & $6,3(0,2)$ & $6,8(0,2)$ & $4,3(0,3)$ & $7,1(0,5)$ & $5,3(0,3)$ & $5,8(0,3)$ \\
\hline Tous les âges & $14,8(0,7)$ & $13,5(0,6)$ & $12,9(0,7)$ & $13,4(0,6)$ & $12,1(0,7)$ & $11,9(0,7)$ & $10,3(0,6)$ & $10,1(0,6)$ \\
\hline
\end{tabular}

${ }^{1}$ Effet secondaire suivant l'immunisation (ESSI)

${ }^{2}$ Effet indésirable grave (EIG)

La baisse observée dans les déclarations de cas sur l'ensemble de la population et par groupe d'âge est probablement due à une combinaison de facteurs liés aux changements apportés aux programmes d'immunisation financés par l'État ainsi qu'aux lignes directrices régissant la déclaration des ESSI. Plusieurs provinces et territoires ont lancé des programmes de vaccination universelle contre la varicelle et par le vaccin conjugué contre le méningocoque pour les nourrissons, entre 2004 et 2006, et des programmes de vaccination contre le VPH (virus du papillome humain) pour les filles d'âge scolaire, entre 2007 et 2010 . Plusieurs campagnes 
de rattrapage, pour divers groupes d'âge, ont également été menées, dont la plupart ont pris fin en 2011 ou 2012. En outre, un certain nombre d'autorités provinciales n'encouragent plus la déclaration des effets les plus légers, telles que la plupart des réactions au point d'administration du vaccin. II est important de souligner également que les déclarations concernant les vaccins administrés les années antérieures à 2012 ont eu plus de temps à parvenir au système de surveillance que celles concernant les vaccins administrés en 2012. Le choix du 30 avril comme date limite d'inclusion des déclarations repose sur l'observation que plus de $80 \%$ des déclarations relatives aux vaccins administrés au cours d'une année civile donnée ont déjà été reçues à quatre mois de la fin de l'année. Les chiffres de l'année 2012 seront mis à jour dans le cade du rapport annuel pour les vaccins administrés en 2013.

Un total de 46481347 doses de vaccin ont été distribuées au Canada en 2011 et 2012, ce qui donne des taux de déclaration pour 100000 doses distribuées de 15,2 pour tous les ESSI et de 0,85 pour les EIG.

\section{Sources de déclaration}

Les figures 1A et 1B illustrent la répartition des sources de déclaration des ESSI et des EIG, stratifiées par groupe d'âge, relativement aux vaccins administrés de 2005 à 2012. En particulier, en ce qui a trait aux enfants âgés de moins de 18 ans, le réseau IMPACT a présenté, en moyenne, 4,1\% de l'ensemble des déclarations des ESSI, mais a généré $54,3 \%$ de tous les EIG. On remarque une tendance claire dans la chute du nombre de déclarations par un détenteur d'une autorisation de mise en marché (DAMM), à la suite de la modification apportée en 2011 à la réglementation en matière de déclaration. De 2005 à 2010, les déclarations par un DAMM comptaient pour 9,8 \% de toutes les déclarations d'ESSI et pour 7,1\% de celles d'EIG. En 2011 et 2012, la contribution relative est descendue à $6,0 \%$ de toutes les déclarations d'ESSI et à $4,3 \%$ de celles d'EIG. Étant donné le volume de déclarations des programmes fédéraux, provinciaux et territoriaux et du réseau IMPACT, ce changement n'a pas eu d'effet important sur les tendances en matière de déclaration du Système canadien de surveillance des effets secondaires suivant l'immunisation (SCSESSI); en outre, les déclarations sont recueillies et étudiées par la Direction des produits de santé commercialisés de Santé Canada et les renseignements ne sont donc pas perdus.

Figure $1 \mathrm{~A}$ : Sources de déclaration des $\mathrm{ESSI}^{1}$ pour les enfants et les adultes par année d'administration des vaccins, de 2005 à 2012

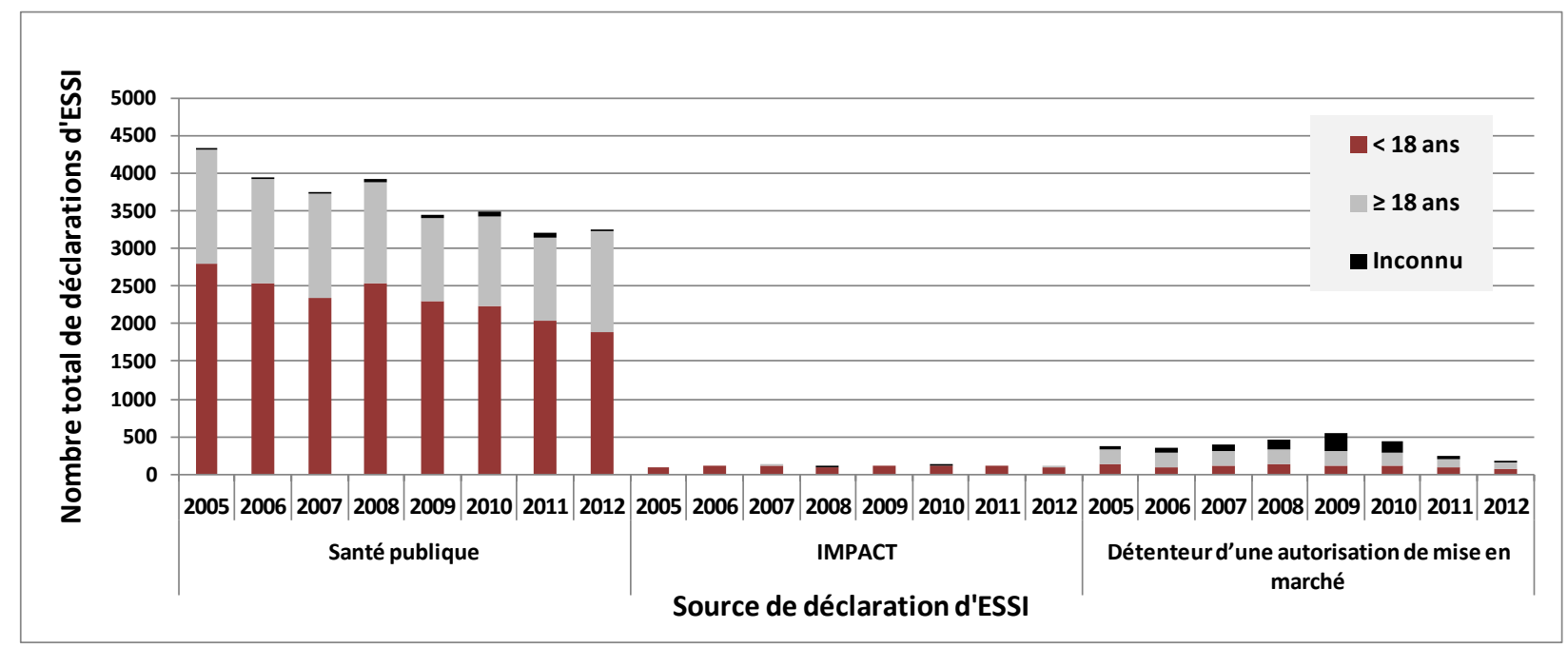

${ }^{1}$ Effet secondaire suivant l'immunisation (ESSI) 


\section{Figure 1B : Sources de déclaration des $\mathrm{ESSI}^{1}$ graves $(\mathrm{EIG})^{2}$ pour les enfants et les adultes par année} d'administration des vaccins, de 2005 à 2012

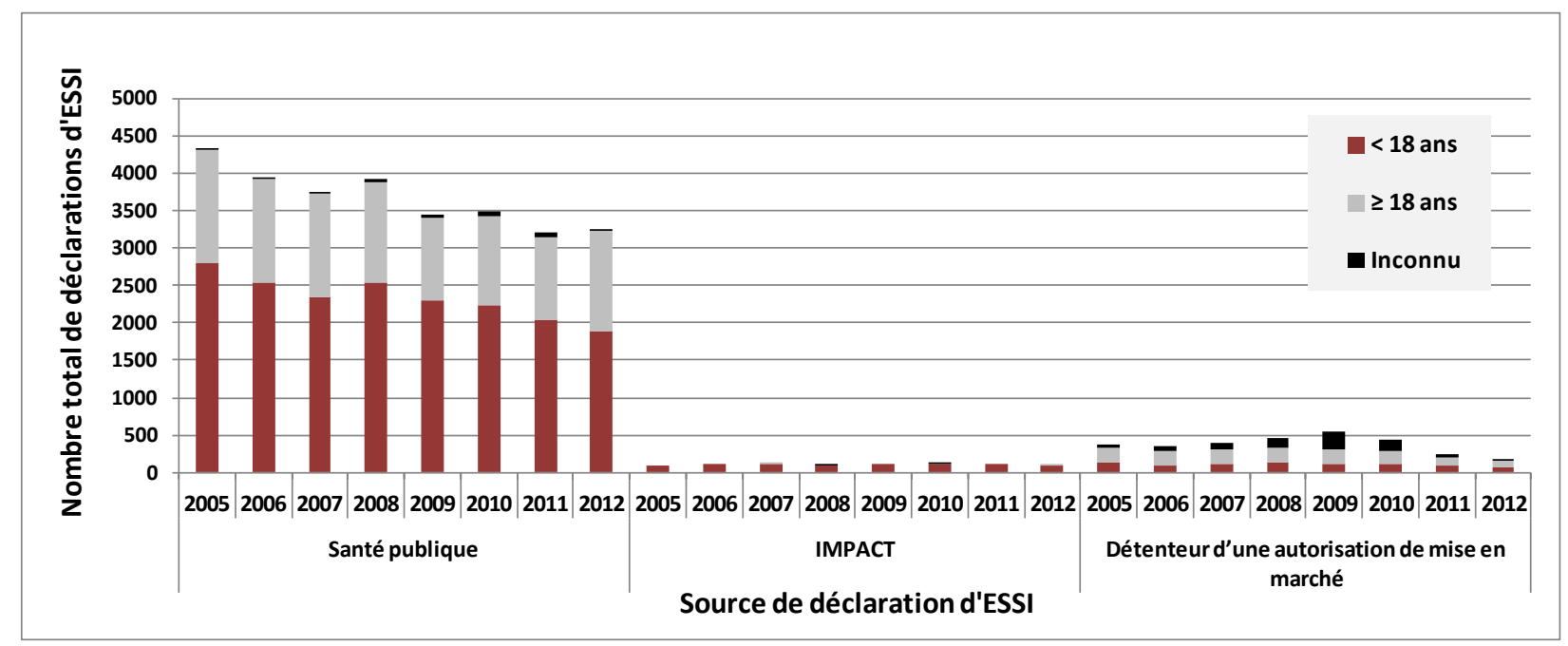

${ }^{1}$ Effet secondaire suivant l'immunisation (ESSI)

${ }^{2}$ Effet indésirable grave (EIG)

\section{Répartition par âge et par sexe}

En ce qui concerne les vaccins administrés en 2012, la répartition par âge parmi les déclarations d'ESSI (EIG) était : $<1$ an : $14 \%$ de tous les ESSI (24\% de tous les EIG); de 1 à < 2 ans : $16 \%$ (32\%); de 2 à < 7 ans : $14 \%$ (12\%); de 7 à < 18 ans : $14 \%$ (9\%); de 18 à < 65 ans : $32 \%$ (14\%); 65 ans et plus : $9 \%$ (8\%); âge inconnu : $1 \%(1 \%)$. La répartition est demeurée assez constante avec le temps (données non représentées). La plus grande proportion de cas graves signalés parmi les jeunes enfants reflète la contribution de la surveillance syndromique active par IMPACT.

En ce qui concerne le sexe, le rapport femmes-hommes est demeuré assez constant, relativement aux vaccins administrés de 2005 à 2012, chez les enfants âgés de moins de 7 ans (intervalle de 0,8 à 0,9 ) et chez tous les adultes (intervalle de 3,4 à 4,1). En revanche, ce rapport a constamment augmenté chez les enfants âgés de 7 à < 18 ans, pour passer de 1,1 en 2005 à 1,6 en 2007; il a atteint un sommet de 2,3 en 2011, avant de chuter à 1,8 en 2012. Les changements correspondaient au lancement des programmes universels de vaccination des femmes contre le virus du papillome humain (VPH), ayant commencé en Ontario en 2007, puis s'étant étendu dans toutes les autres provinces en 2008, ainsi que dans les territoires en 2010, en plus des programmes de rattrapage destinés aux filles d'âge scolaire plus âgées, qui se sont terminés avant 2012.

\section{Soins de santé utilisés et résultats relatifs aux ESSI des vaccins administrés en 2012}

La répartition des soins de santé associés utilisés était : aucun dans $24,5 \%$ des cas; conseil d'un professionnel de la santé dans $5 \%$ des cas; examen médical non urgent dans $35,9 \%$ des cas; examen médical urgent dans $17,9 \%$ des cas; hospitalisation dans $5,2 \%$ des cas; prolongation d'une hospitalisation déjà en cours dans $0,1 \%$ des cas et soin inconnu dans $11,4 \%$ des cas signalés. La seule tendance temporelle observée en matière de déclaration était le fait que l'utilisation était précisée dans une proportion croissante de déclarations avec le temps, avec de légères hausses des proportions d'examens médicaux urgents et non urgents (données non représentées).

La répartition de l'issue était : $67,8 \%$ des cas signalés étaient complètement rétablis; $16,2 \%$ n'étaient pas encore rétablis; $0 \%$ souffraient d'une incapacité permanente; $0,1 \%$ des cas s'était soldé par un décès; enfin, on ignorait 
l'issue de $15,8 \%$ des cas signalés. On n'a observé aucune tendance temporelle notable, relativement aux vaccins administrés de 2005 à 2012 (données non représentées). Des issues mortelles et des cas d'incapacité permanente sont rarement signalés (intervalle annuel de 5 à 14 et de 0 à 11 du nombre total de déclarations, respectivement).

\section{Effets indésirables signalés}

Le tableau 4 montre la distribution par Classe par système et organe de MedDRA pour tous les ESSI signalés, relativement à tous les vaccins administrés en 2012, ainsi que les répartitions comparatives relatives aux vaccins administrés en 2011 et de 2005 à 2010. La seule classe pour laquelle on observe une augmentation temporelle constante mesurable du nombre de cas est celle du système immunitaire, dont la proportion est passée de 5,8 \% des vaccins administrés de 2005 à 2010 , à $8,9 \%$ en 2011, puis à $14,0 \%$ en 2012. Les 490 cas signalés à la suite de vaccins administrés en 2012 étaient tous associés à des termes privilégiés relatifs à des ESSI allergiques (essentiellement l'hypersensibilité, certaines réactions anaphylactiques et deux réactions immunitaires de type III); seulement 11 (2,2 \%) étaient graves. Lors d'une comparaison croisée avec la classification des principaux ESSI, la majorité des cas tombait sous les catégories : autre réaction allergique (198), éruption cutanée seule (153), réaction anaphylactique (50) ou réaction au point de vaccination (43). On a signalé un seul cas d'arthrite, un d'érythème polymorphe, un de maladie sérique et aucun de troubles immuns sujets de préoccupations. Les déclarations de réactions anaphylactiques se répartissaient dans plusieurs groupes d'âge et vaccins, sans grappe liée à un lot.

Tableau 4 : Répartition selon la Classe par système et organe de tous les ESSI ${ }^{1}$ et les EIG $^{2}$ signalés relatifs à des vaccins administrés de 2005 à 2012 (le pourcentage moyen de distribution est montré pour les années 2005 à 2010)

\begin{tabular}{|c|c|c|c|}
\hline Classe par système et organe & \multicolumn{3}{|c|}{$\begin{array}{c}\% \text { des ESSI signalés mentionnant au moins } \\
1 \text { terme de } \mathrm{CSO}^{3}\end{array}$} \\
\hline Affections hématologiques et du système lymphatique & 2,1 & 2,7 & $\overline{1,9}$ \\
\hline Affections congénitales, familiales et génétiques & 0 & 0 & 0 \\
\hline Affections de l'oreille et du labyrinthe & 0,6 & 0,9 & 1,1 \\
\hline Affections gastro-intestinales & 14,9 & 19,3 & 20,2 \\
\hline Troubles généraux et anomalies au site d'administration & 61,2 & 66,6 & 66,7 \\
\hline Affections hépatobiliaires & 0,1 & 0,1 & 0,1 \\
\hline Affections du système immunitaire & 5,8 & 8,9 & 14,0 \\
\hline Infections et infestations & 7,0 & 10,1 & 11,5 \\
\hline Affections musculo-squelettiques et du tissu conjonctif & 8,4 & 10,6 & 10,3 \\
\hline Tumeurs bénignes, malignes et non précisées (y compris les kystes et polypes) & 0 & 0 & 0 \\
\hline Affections du système nerveux & 19,5 & 20,4 & 21,6 \\
\hline Affections gravidiques, puerpérales et périnatales & 0,1 & 0 & 0 \\
\hline Troubles psychiatriques & 2,6 & 2,2 & 2,1 \\
\hline Affections du rein et des voies urinaires & 0,2 & 0,3 & 0,4 \\
\hline
\end{tabular}




\begin{tabular}{|c|c|c|c|}
\hline Affections des organes de reproduction et du sein & 0,2 & 0,1 & 0,3 \\
\hline Affections respiratoires, thoraciques et médiastinales & 9,3 & 11,8 & $\overline{11,4}$ \\
\hline Affections de la peau et du tissu sous-cutané & 35,2 & 40,0 & 37,6 \\
\hline Actes médicaux et chirurgicaux & 0,1 & 0,1 & $\overline{0}$ \\
\hline Affections vasculaires & 3,1 & 3,4 & 4,2 \\
\hline
\end{tabular}

${ }^{1}$ Effet secondaire suivant l'immunisation (ESSI)

${ }^{2}$ Effet indésirable grave (EIG)

${ }^{3}$ Chaque déclaration d'ESSI peut contenir plusieurs termes relatifs aux ESSI appartenant à différentes Classes par système et organe; le total pour chaque période de vaccination peut donc excéder $100 \%$ des cas signalés.

\section{Principale raison de déclaration}

Le tableau 5 montre les résultats de la classification de l'examen du dossier médical des 3508 cas d'ESSI signalés après l'administration de vaccins effectuée en 2012, y compris, pour chaque catégorie principale, les taux d'ESSI et d'EIG signalés pour 100000 doses distribuées et la proportion des types précis d'ESSI.

\section{Tableau 5 : Répartition, gravité et fréquence de déclaration des $\mathrm{ESSI}^{1}$, en fonction de l'examen des dossiers médicaux}

\begin{tabular}{|c|c|c|c|c|c|}
\hline \multirow[t]{2}{*}{$\begin{array}{c}\text { Catégorie d'effets } \\
\text { secondaires suivant } \\
\text { l'immunisation (ESSI) }\end{array}$} & \multirow{2}{*}{$\begin{array}{c}\text { Total des } \\
\text { ESSI/Effets } \\
\text { indésirables } \\
\text { graves (EIG) }\end{array}$} & \multicolumn{2}{|c|}{$\begin{array}{c}\text { Taux de déclaration } \\
\text { pour } 100000 \text { doses } \\
\text { distribuées }\end{array}$} & \multirow[t]{2}{*}{$\begin{array}{c}\text { Répartition par catégorie } \\
\text { selon } \\
\text { le type précis d'ESSI }\end{array}$} & \multirow[t]{2}{*}{$\begin{array}{l}\text { \% type } \\
\text { d'EIG }\end{array}$} \\
\hline & & Tous les cas & EIG & & \\
\hline \multirow{5}{*}{$\begin{array}{l}\text { Réactions au site de } \\
\text { vaccination ou à proximité }\end{array}$} & \multirow[t]{5}{*}{$1238 / 20$} & \multirow[t]{5}{*}{5,25} & \multirow[t]{5}{*}{0,08} & Abcès : $1,5 \%$ & 5 \\
\hline & & & & Cellulite : $17 \%$ & 7 \\
\hline & & & & OEdème sévère d'un membre : 14 \% & 2 \\
\hline & & & & $\begin{array}{l}\text { Douleur au membre vacciné d'une } \\
\text { durée }>7 \text { jours : } 1,5 \%\end{array}$ & 0 \\
\hline & & & & Autre réaction locale : $66 \%$ & 0,1 \\
\hline \multirow{3}{*}{$\begin{array}{l}\text { Événements allergiques ou } \\
\text { de type allergique }\end{array}$} & \multirow[t]{3}{*}{$443 / 10$} & \multirow[t]{3}{*}{1,88} & \multirow[t]{3}{*}{0,04} & Réaction anaphylactique : $11,7 \%$ & 12 \\
\hline & & & & Syndrome oculo-respiratoire : $8,6 \%$ & 3 \\
\hline & & & & $\begin{array}{l}\text { Autre manifestation allergique : } \\
79,7 \%\end{array}$ & 0,5 \\
\hline \multirow[t]{8}{*}{ Événements neurologiques } & \multirow[t]{8}{*}{$147 / 64$} & \multirow[t]{8}{*}{0,62} & \multirow[t]{8}{*}{0,27} & Encéphalomyélite : $6,1 \%$ & 67 \\
\hline & & & & Ataxie/cérébellite : 0,7\% & 100 \\
\hline & & & & Méningite à liquide clair : 1,4 \% & 100 \\
\hline & & & & Syndrome de Guillain-Barré : 3,4 \% & 80 \\
\hline & & & & Paralysie de Bell : 9,5\% & 7 \\
\hline & & & & $\begin{array}{l}\text { Autre paralysie d'une durée } \\
>1 \text { jour : } 1,4 \%\end{array}$ & 50 \\
\hline & & & & Convulsions : $67,3 \%$ & 43 \\
\hline & & & & $\begin{array}{l}\text { Autre manifestation neurologique : } \\
10,2 \%\end{array}$ & 40 \\
\hline Éruption cutanée seulement & $694 / 0$ & 2,94 & 0 & & \\
\hline \multirow{7}{*}{$\begin{array}{l}\text { Autres événements indiqués } \\
\text { sur le formulaire de } \\
\text { déclaration du SCSESSI }\end{array}$} & \multirow[t]{7}{*}{$153 / 21$} & \multirow[t]{7}{*}{0,65} & \multirow[t]{7}{*}{0,09} & Arthrite : $11,8 \%$ & 0 \\
\hline & & & & $\begin{array}{l}\text { Épisode hypotonique-hyporéactif : } \\
19,6 \%\end{array}$ & 33 \\
\hline & & & & Intussusception : 2,6 \% & 50 \\
\hline & & & & Paresthésie : $41,8 \%$ & 0 \\
\hline & & & & Parotidite : $5,2 \%$ & 0 \\
\hline & & & & Pleurs persistants : $11,1 \%$ & 0 \\
\hline & & & & Thrombocytopénie : 7,8 \% & 75 \\
\hline \multirow{4}{*}{$\begin{array}{l}\text { Autres effets généraux non } \\
\text { précisés sur le formulaire de } \\
\text { déclaration du SCSESSI }\end{array}$} & \multirow[t]{4}{*}{$390 / 52$} & \multirow[t]{4}{*}{1,65} & \multirow[t]{4}{*}{0,22} & Fièvre uniquement : $14,9 \%$ & 7 \\
\hline & & & & Infection : $22,9 \%$ & 20 \\
\hline & & & & Syndrome pseudogrippal : $7,7 \%$ & 3 \\
\hline & & & & Autres symptômes généraux : 28 \% & 7 \\
\hline
\end{tabular}




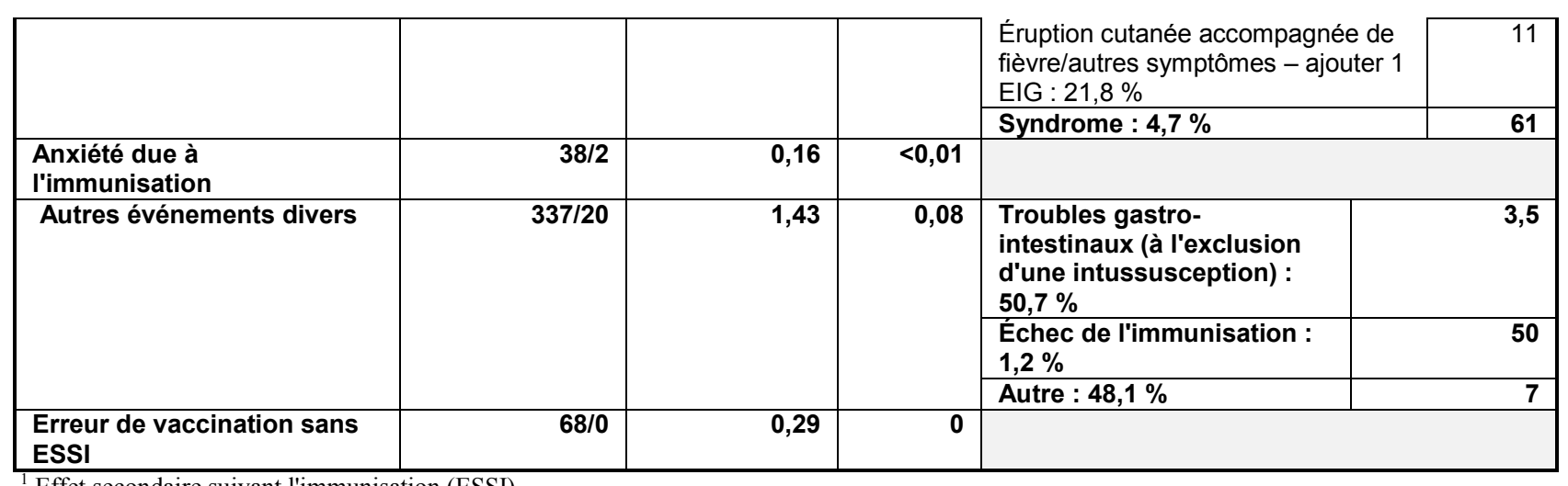

${ }^{1}$ Effet secondaire suivant l'immunisation (ESSI)

Le tableau 6 montre la répartition en fonction de la gravité et du groupe d'âge dans les catégories des principaux ESSI. Les réactions au point de vaccination ou près de celui-ci et les éruptions cutanées seules comptaient pour $55,1 \%$ de toutes les déclarations reçues, relativement aux vaccins administrés en 2012, mais seulement pour $11,2 \%$ des EIG signalés. En revanche, les manifestations neurologiques comptaient pour seulement $4,2 \%$ de toutes les déclarations de 2012, mais pour $43,5 \%$ des EIG signalés. Le réseau IMPACT, qui recherche activement les manifestations neurologiques, a signalé 45 (70\%) des 64 manifestations neurologiques graves.

Tableau 6 : Profils des catégories des principaux ESSI par gravité et groupe d'âge

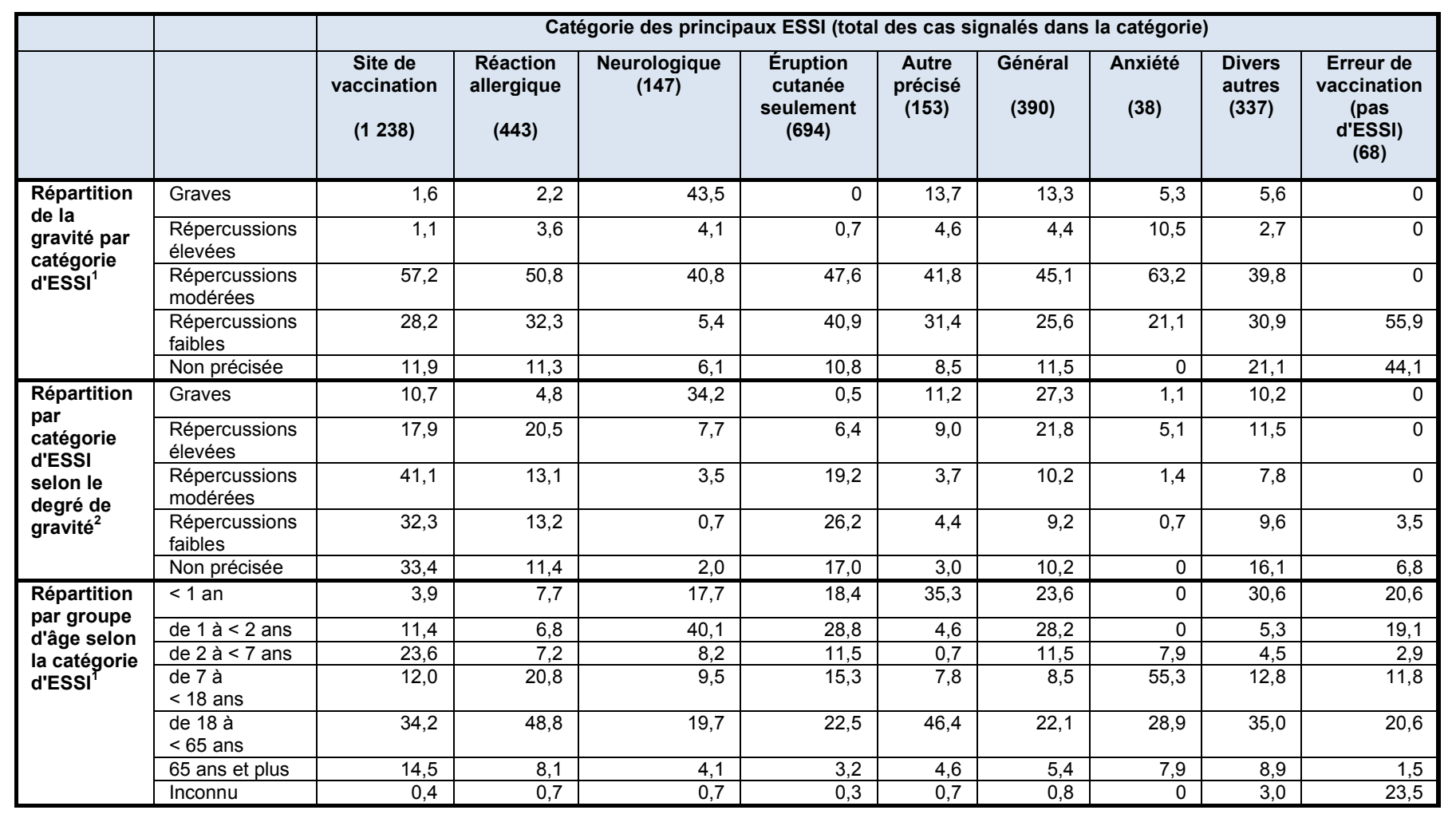




\begin{tabular}{|c|c|c|c|c|c|c|c|c|c|c|}
\hline \multirow{7}{*}{$\begin{array}{l}\text { Répartition } \\
\text { par } \\
\text { catégorie } \\
\text { d'ESSI } \\
\text { selon le } \\
\text { groupe } \\
\text { d'âge }^{2}\end{array}$} & $<1$ an & 9,6 & 6,8 & 5,2 & 25,7 & 10,8 & 18,4 & 0 & 20,6 & 2,8 \\
\hline & de 1 à $<2$ ans & 24,4 & 5,2 & 10,2 & 34,6 & 1,2 & 19,0 & 0 & 3,1 & 2,2 \\
\hline & de 2 à $<7$ ans & 60,6 & 6,6 & 2,5 & 16,6 & 0,2 & 9,3 & 0,6 & 3,1 & 0,4 \\
\hline & $\begin{array}{l}\text { de } 7 \text { à } \\
<18 \text { ans }\end{array}$ & 31,2 & 19,2 & 2,9 & 22,2 & 2,5 & 6,9 & 4,4 & 9,0 & 1,7 \\
\hline & $\begin{array}{l}\text { de } 18 \text { à } \\
<65 \text { ans }\end{array}$ & 37,7 & 19,2 & 2,6 & 13,9 & 6,3 & 7,6 & 1,0 & 10,5 & 1,2 \\
\hline & 65 ans et plus & 58,7 & 11,8 & 2,0 & 7,2 & 2,3 & 6,9 & 1,0 & 9,8 & 0,3 \\
\hline & Inconnu & 35,3 & 12,6 & 4,2 & 19,8 & 4,4 & 11,1 & 1,1 & 9,6 & 1,9 \\
\hline
\end{tabular}

${ }^{1}$ Les totaux des colonnes sont de $100 \%$ pour chaque catégorie des principaux ESSI.

${ }^{2}$ Les totaux des rangées sont de $100 \%$ pour le degré de gravité ou le groupe d'âge.

Les enfants âgés de 1 à < 2 ans comptaient pour la plus grande proportion des déclarations dans les catégories des principaux ESSI que sont les réactions neurologiques, les éruptions cutanées seules et les réactions générales. Les enfants âgés de 7 à $<18$ ans comptaient pour $55,3 \%$ des réactions d'anxiété due à la vaccination. Les adultes âgés de 18 à $<65$ ans formaient le groupe d'âge le plus important, relativement aux catégories des principaux ESSI que sont les réactions allergiques, au site de vaccination, autres précisées et diverses. Dans chaque catégorie d'âge, les groupes des principaux ESSI les plus souvent signalés étaient les réactions au site de vaccination, chez tous les cas âgés de 2 ans et plus, et les éruptions cutanées seules, chez les cas âgés de moins de 2 ans.

\section{Vaccins administrés en 2012}

Le tableau 7 comporte la liste des vaccins pour lesquels au moins une déclaration d'ESSI a été reçue, en ce qui concerne les vaccins administrés au cours de l'année civile 2012, regroupés par contenu antigénique, ainsi qu'en fonction du fait qu'ils étaient inclus ou non dans des programmes d'immunisation du Canada financés par l'État pour administration systématique ou limitée ou qu'ils étaient vendus principalement dans le marché privé. Le tableau fournit des données sur chaque groupe antigénique à propos de la proportion des déclarations si un seul vaccin avait été administré, du nombre total de déclarations d'ESSI et d'EIG à la suite de vaccins administrés en 2012, ainsi que du taux de déclaration combiné de 2011 et 2012 pour 100000 doses distribuées. On doit interpréter avec prudence la variation des taux de déclaration. Par exemple, le taux observé avec le vaccin dcaTVPI-HB-Hib est presque quatre fois celui observé avec le vaccin dcaT-VPI-Hib, tous les deux administrés aux nourrissons aux âges de 2, 4 et 6 mois. Le produit contenant le HB est administré principalement en ColombieBritannique, dont le taux de déclaration est considérablement plus élevé que celui des autres provinces (données non représentées). En outre, les renseignements concernant les doses distribuées ne tiennent pas compte des doses retournées ou gaspillées et, au mieux, il s'agit d'une mauvaise valeur de substitution pour les taux de déclaration relatifs aux vaccins administrés, taux qui ne sont actuellement pas disponibles partout au Canada.

\section{Tableau 7 : Abréviations et groupes de vaccins servant au rapport annuel ${ }^{1}$}

\begin{tabular}{|c|c|c|c|c|c|c|}
\hline Infections ciblées & $\begin{array}{c}\text { Nom sous lequel le } \\
\text { vaccin est } \\
\text { commercialisé au } \\
\text { Canada (DAMM }{ }^{2} \text { ) }\end{array}$ & $\begin{array}{l}\text { Abréviation } \\
\text { du groupe } \\
\text { de vaccins }\end{array}$ & $\begin{array}{c}\% \text { seul } \\
\text { vaccin } \\
\text { administré }\end{array}$ & $\begin{array}{l}2012 \\
\text { ESSI }\end{array}$ & $\begin{array}{c}2012 \\
\text { EIG } \\
(\% \\
\text { ESSI) }\end{array}$ & $\begin{array}{c}\text { Taux de } \\
\text { déclaration } \\
\text { d'ESSI } \\
\text { combinés de } \\
2011-2012 \text { pour } \\
100000 \text { doses } \\
\text { distribuées }\end{array}$ \\
\hline \multicolumn{7}{|c|}{ Vaccins administrés en vertu de programmes d'immunisation financés par l'État } \\
\hline Diphtérie, tétanos, coqueluche & $\begin{array}{l}\text { Adacel® (SP) } \\
\text { Boostrix }^{\mathrm{TM}} \text { (GSK) }\end{array}$ & dcaT & 67 & 334 & $3(0.9)$ & 20.0 \\
\hline \multirow[t]{2}{*}{$\begin{array}{l}\text { Diphtérie, tétanos, } \\
\text { coqueluche, Polio }\end{array}$} & $\begin{array}{l}\text { Quadracel } \circledast(S P) \\
\text { Infanrix }{ }^{T M} \text {-IPV (GSK) }\end{array}$ & DCaT-VPI & 53 & 176 & $6(3.4)$ & 101.0 \\
\hline & $\begin{array}{l}\text { Adacel }^{\circledR} \text {-Polio (SP), } \\
\text { Boostrix®-Polio (GSK) }\end{array}$ & dcaT-VPI & 57 & 60 & $1(1.7)$ & 15.2 \\
\hline
\end{tabular}




\begin{tabular}{|c|c|c|c|c|c|c|}
\hline $\begin{array}{l}\text { Diphtérie, tétanos, } \\
\text { coqueluche, } \\
\text { Polio, H. influenzae de type b }\end{array}$ & $\begin{array}{l}\text { Pediacel® (SP), } \\
\text { Infanrix }{ }^{T M}-I P V / H i b \\
\text { (GSK), }\end{array}$ & $\begin{array}{l}\text { DCaT-VPI- } \\
\text { Hib }\end{array}$ & 42 & 498 & $38(7.6)$ & 37.6 \\
\hline $\begin{array}{l}\text { Diphtérie, tétanos, } \\
\text { coqueluche, } \\
\text { Polio, H. influenzae de type b, } \\
\text { hépatite B }\end{array}$ & Infanrix hexa ${ }^{\mathrm{TM}}$ (GSK) & $\begin{array}{l}\text { DCaT-HB- } \\
\text { VPI-Hib }\end{array}$ & 16 & 214 & $12(5.6)$ & 148.2 \\
\hline Diphtérie, tétanos, polio & $\begin{array}{l}\text { Td Polio Adsorbées } \\
\text { (SP) }\end{array}$ & Td-VPI & 47 & 15 & $1(6.7)$ & 22.1 \\
\hline Diphtérie, tétanos & Td Adsorbées (SP) & $\mathrm{Td}$ & 54 & 80 & $2(2.5)$ & 10.3 \\
\hline \multirow[t]{2}{*}{ Pneumococcie invasive } & $\begin{array}{l}\text { Prevnar® (Pfiz), } \\
\text { Synflorix }{ }^{\top M} \text { (GSK), } \\
\text { Prevnar®13 (Pfiz) }\end{array}$ & Pneu-C & 6 & 682 & $78(11.4)$ & 56.8 \\
\hline & $\begin{array}{l}\text { Pneumo® } 23(\mathrm{SP}) \\
\text { Pneumovax® } 23 \text { (MF) }\end{array}$ & Pneu-P-23 & 50 & 228 & 18(7.9) & 38.0 \\
\hline \multirow[t]{3}{*}{ Méningococcie invasive } & $\begin{array}{l}\text { Meningitec }^{\circledR}(\text { Pfiz), } \\
\text { Menjugate }{ }^{\circledR}(\mathrm{NP}), \\
\text { Neis Vac-C } C^{\circledR} \text { (GSK) }\end{array}$ & Men-C-C & 6 & 468 & $61(13.0)$ & 85.2 \\
\hline & $\begin{array}{l}\text { Menactra }^{\Theta} \text { (SP), } \\
\text { Menveo }^{\mathrm{TM}} \text { (NVD), } \\
\text { Nimenrix }^{\mathrm{TM}} \text { (GSK) }\end{array}$ & $\begin{array}{l}\text { Men-C- } \\
\text { ACYW-135 }\end{array}$ & 48 & 84 & $2(2.4)$ & 23.7 \\
\hline & $\begin{array}{l}\text { Menomune } \AA- \\
\mathrm{A} / \mathrm{C} / \mathrm{Y} / \mathrm{W}-135(\mathrm{SP})\end{array}$ & $\begin{array}{l}\text { Men-P- } \\
\text { ACYW-135 }\end{array}$ & 0 & 2 & 0 & $\mathrm{NC}^{3}$ \\
\hline Hépatite $B$ & $\begin{array}{l}\text { Engerix }{ }^{\circledR}-\mathrm{B}(\mathrm{GSK}) \text {, } \\
\text { Recombivax } \mathrm{HB} \otimes \\
\text { (MF) }\end{array}$ & $\mathrm{HB}$ & 38 & 213 & $11(5.2)$ & 22.0 \\
\hline Hépatite $A$, hépatite $B$ & $\begin{array}{l}\text { Twinrix }{ }^{\circledR} \text { et Twinrix }{ }^{\circledR} \\
\text { Junior (both GSK) }\end{array}$ & HAHB & 56 & 90 & $3(3.3)$ & 10.1 \\
\hline Hépatite $A$ & $\begin{array}{l}\text { Avaxim } ® \text { et Avaxim } ®- \\
\text { Pediatric (SP), } \\
\text { Havrix®1440 \& } \\
\text { Havrix®720Junior } \\
\text { (GSK), Vaqta } ®(M F)\end{array}$ & $\mathrm{HA}$ & 24 & 70 & $2(2.9)$ & 14.1 \\
\hline Hépatite A, typhoïde & $\operatorname{ViVAXIM}^{\mathrm{TM}}(\mathrm{SP})$ & HA-Typh-I & 46 & 11 & 0 & 20.8 \\
\hline Rougeole, oreillons, rubéole & $\begin{array}{l}\text { M-M-R® II (MF), } \\
\text { Priorix® (GSK) }\end{array}$ & ROR & 20 & 393 & $29(7.4)$ & 68.2 \\
\hline $\begin{array}{l}\text { Rougeole, oreillons, rubéole + } \\
\text { Varicelle }\end{array}$ & Priorix-Tetra" (GSK) & RORV & 17 & 163 & $42(25.8)$ & 53.7 \\
\hline Varicelle & $\begin{array}{l}\text { Varilrix® (GSK), } \\
\text { Varivax® III (MF) }\end{array}$ & Var & 22 & 358 & $16(4.5)$ & 74.0 \\
\hline Grippe & 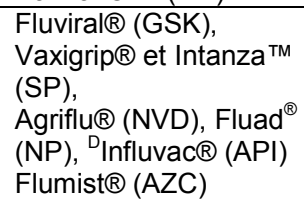 & $\operatorname{lnf}$ & 82 & 913 & $36(3.9)$ & 8.5 \\
\hline Virus du papillome humain & $\begin{array}{l}\text { Gardasil囚 (MF), } \\
\text { Cervarix }^{\mathrm{TM}}(\mathrm{GSK})\end{array}$ & VPH & 65 & 185 & $9(4.9)$ & 29.8 \\
\hline Rotavirus & $\begin{array}{l}\text { Rotarix }{ }^{\mathrm{TM}}(\mathrm{GSK}) \\
\text { RotaTeq }{ }^{\circledR}(\mathrm{MF})\end{array}$ & Rota & 11 & 228 & $21(9.2)$ & 30.5 \\
\hline \multicolumn{7}{|c|}{ Vaccins à usage particulier (financés par l'État dans certaines situations) } \\
\hline Tuberculose & BCG (SP) & $\begin{array}{l}\text { Bacille de } \\
\text { Calmette- } \\
\text { Guérin } \\
\text { (BCG) }\end{array}$ & 100 & 5 & 0 & $\mathrm{NC}^{3}$ \\
\hline Rage & $\begin{array}{l}\text { Imovax }{ }^{\circledR} \text { Rabies(SP), } \\
\text { RabAvert } \circledast \text { (NP) }\end{array}$ & Rage & 85 & 39 & 0 & 135.1 \\
\hline
\end{tabular}




\begin{tabular}{|c|c|c|c|c|c|c|}
\hline \multicolumn{7}{|l|}{ Vaccins non financés par l'État } \\
\hline Zona & Zostavax® (MF) & Zona & 91 & 80 & $2(2.5)$ & 27.7 \\
\hline H. influenzae de type b & $\begin{array}{l}\text { ACT-HIB® (SP), } \\
\text { Hiberix }^{\circledR}(\mathrm{GSK}) \text {, Liquid } \\
\text { PedvaxHib®(MF) }^{-}\end{array}$ & $\mathrm{Hib}$ & 6 & 16 & $3(18.8)$ & 63.8 \\
\hline Polio & Imovax® Polio (SP) & VPI & 3 & 29 & 0 & 28.2 \\
\hline Choléra & Dukoral $^{(8}(\mathrm{CV})$ & Chol-Ecol-O & 77 & 26 & $1(3.8)$ & 10.5 \\
\hline Encéphalite japonaise & IXIARO® (NP) & EJ & 57 & 7 & $1(14.3)$ & $\mathrm{NC}^{3}$ \\
\hline $\begin{array}{l}\text { Encéphalite transmise par les } \\
\text { tiques }\end{array}$ & $\begin{array}{l}\text { FSME-IMMUN } \\
\text { (Bax) }\end{array}$ & ETT & 100 & 1 & 0 & $\mathrm{NC}^{3}$ \\
\hline Typhoïde & $\begin{array}{l}\text { Typherix }{ }^{\text {II" }}(\text { GSK }), \\
\text { Typhim Vi® }(\mathrm{SP}), \\
\text { Vivotif } ®(\mathrm{CV})\end{array}$ & Typh-I/O & 42 & 43 & $1(2.3)$ & 18.4 \\
\hline Fièvre jaune & YF-Vax® (SP) & FJ & 24 & 25 & 0 & 33.5 \\
\hline
\end{tabular}

${ }^{1}$ Remarque : Seuls les vaccins commercialisés au Canada pour lesquels au moins une déclaration d'ESSI avait été reçue en date du 30 avril 2013 , en ce qui concerne les vaccins administrés en 2012.

${ }^{2}$ Détenteurs d'une autorisation de mise en marché, notamment : API - Laboratoires Abbott, Limitée; AZC - AstraZeneca Canada Inc.; Bax -

Baxter Corporation; CV - Crucell Vaccines Canada; GSK - GlaxoSmithKline Inc.; MF - Merck Canada Inc.; NP - Novartis Pharmaceuticals Canada Inc.;

NVD - Novartis Vaccines and Diagnostics, Inc.; Pfiz - Pfizer Canada Inc.; SP - Sanofi Pasteur Ltée.

${ }^{3} \mathrm{NC}-$ non calculé en raison de la taille restreinte de l'échantillon $(<10$ déclarations en deux ans).

La figure 2 montre la répartition totale des principaux ESSI par groupe de vaccins. Les réactions au site d'injection ou près de celui-ci comptaient pour plus de $50 \%$ des ESSI signalés relatifs aux vaccins dcaT-VPI, dcaT-VPI, dT, Pneu-P-23 et Zona. Les manifestations neurologiques comptaient pour $24 \%$ de l'ensemble des déclarations relatives au vaccin contre la rougeole, la rubéole, les oreillons et la varicelle (RORV), mais pour une proportion beaucoup plus petite des déclarations relatives aux autres vaccins, notamment le vaccin contre la rougeole, la rubéole et les oreillons (RRO).

\section{Figure 2 : Répartition des principaux effets secondaires suivant l'immunisation (ESSI) par groupe de vaccins}

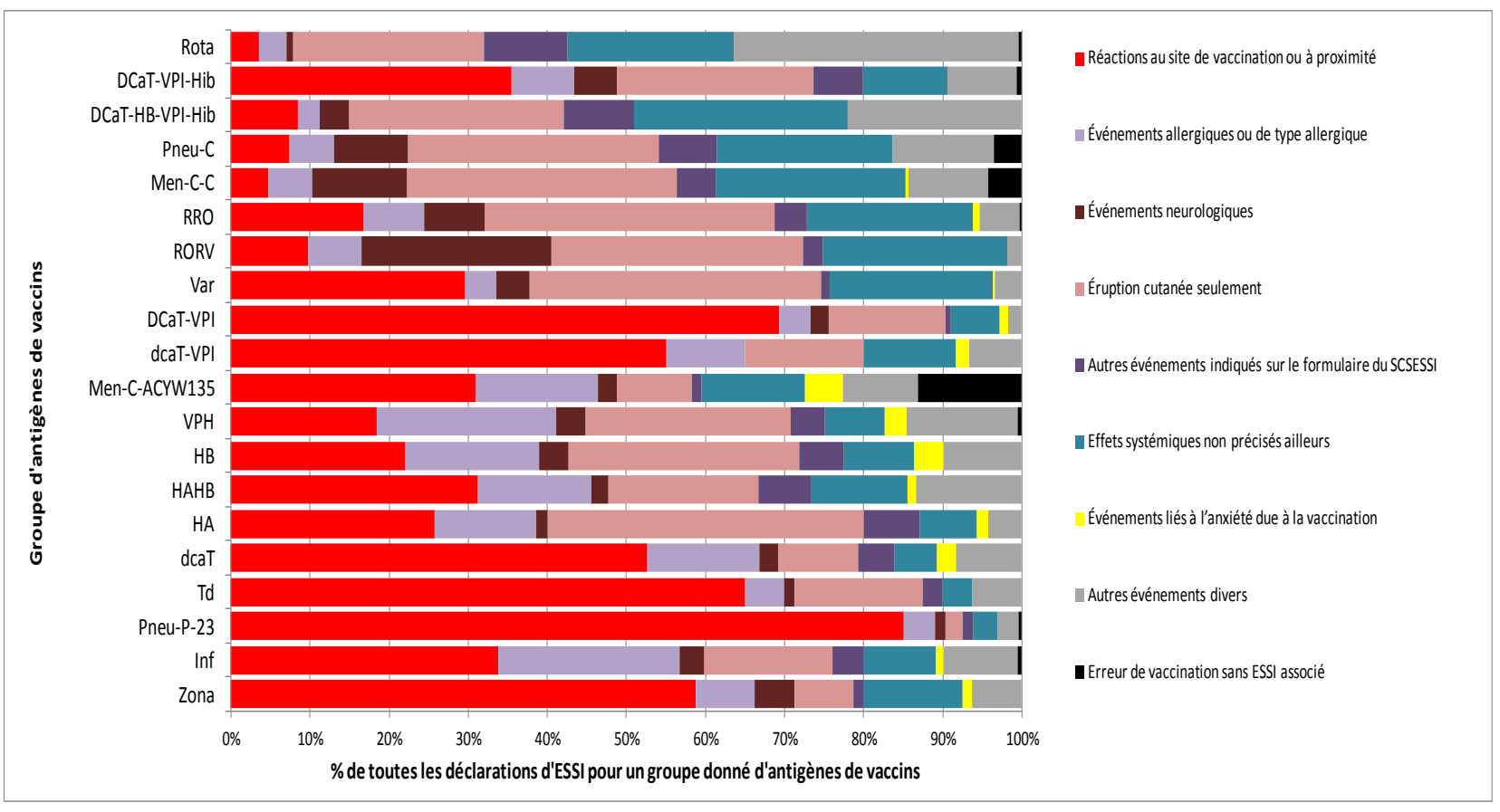




\section{Effets indésirables graves par raison principale de la déclaration}

Parmi les 188 ESSI graves signalés, relativement à des vaccins administrés en 2012, la raison pour laquelle les effets ont été jugés graves était une hospitalisation dans 183 cas et le décès dans cinq cas. Aucun des décès n'a été imputé à un vaccin, comme on l'explique plus bas.

Trois décès sont survenus chez des adultes, trois minutes, 35 minutes et cinq jours après l'administration d'un vaccin antigrippal inactivé (deux fabricants différents et un produit non identifié), pour un taux total de déclaration de 0,3 pour 1 million de doses de vaccin antigrippal distribuées. Tous les décès ont été soudains et dus à un arrêt cardiaque; dans tous les cas, on a jugé qu'une cardiopathie préexistante était probablement la cause du décès.

Un décès a suivi l'administration d'une deuxième dose d'un vaccin RRO à un enfant d'âge scolaire (taux de déclaration de 1,45 pour 1 million de doses distribuées). Aucun autre vaccin n'avait été administré. Il y a eu apparition d'une fièvre aiguë dans les 24 heures suivant la vaccination puis, trois jours plus tard, de convulsions fébriles, qui ont progressé vers un état de mal épileptique entraînant un coma résistant. Le décès est survenu plusieurs semaines plus tard. La neuro-imagerie a révélé une anomalie temporale droite en foyer, mais aucune cause n'a été trouvée, malgré des examens approfondis. Le moment de l'apparition de la maladie n'étaye pas un rapport de causalité avec la vaccination.

Enfin, un tout-petit est décédé dans les 24 heures suivant son immunisation avec le vaccin dcaT-VPI-Hib seul (taux de déclaration de 0,7 pour 1 million de doses distribuées). L'enquête du coroner a révélé que le décès était attribuable à une infection respiratoire aiguë.

On a signalé 64 manifestations neurologiques graves, dont 43 cas de convulsions, la plupart fébriles. Dans les 42 cas dont l'issue est connue, 41 personnes se sont complètement rétablies; l'exception était un cas connu de sclérose tubéreuse de Bourneville accompagnée de spasmes infantiles. Six cas ont été déclarés après l'administration d'un seul vaccin (RRO, VPH, antigrippal inactivé, dcaT-VPI-HB-Hib, Var, vaccin combiné hépatite $A$ /hépatite $B$ [HAHB]), et les autres cas se sont déclarés après l'administration de deux vaccins ou plus. Tous les cas, sauf huit, étaient associés aux vaccins RRO ou RORV, habituellement administrés avec des vaccins conjugués contre le pneumocoque ou le méningocoque ou les deux. Le taux de déclaration de cas chez qui l'apparition des effets secondaires est survenue dans l'intervalle de risque attendu de 5 à 12 jours était de 1,08 pour 100000 doses distribuées de vaccins contenant la rougeole avec les vaccins RRO et de 4,83 avec les vaccins RORV. Le risque accru de convulsion fébrile à la suite de l'administration d'un vaccin RORV, par rapport à un vaccin $\mathrm{RRO}$, est désormais bien connu et a été récemment documenté au Canada (42).

Les 21 autres manifestations neurologiques graves portent sur plusieurs affections. On a recensé 11 cas possibles de maladie de démyélinisation. On a signalé quatre cas de syndrome de Guillain-Barré, dont trois étant apparu 10, 11 et un nombre inconnu de jours après l'administration de vaccins contre la diphtérie et le tétanos, et un cas apparu 24 heures après l'administration d'un vaccin polysaccharidique contre le pneumocoque. On a signalé deux cas d'encéphalite aiguë : un apparu 11 jours après l'administration d'un vaccin RORV seul et l'autre, 22 jours après l'administration d'un vaccin RORV et de vaccins conjugués contre le pneumocoque et le méningocoque. La causalité n'a été prouvée dans aucun des cas, mais le vaccin vivant atténué du virus aurait pu causer la maladie. Les deux personnes se sont complètement rétablies. On a signalé deux cas d'encéphalomyélite aiguë disséminée : un premier cas s'est manifesté 1 jour après l'administration de la deuxième dose et 29 jours après l'administration de la première dose d'un vaccin contre l'encéphalite japonaise; l'autre est apparu 13 jours après l'administration de la première dose de vaccins contre le VPH et contre l'hépatite $B$. Aucun cas ne répondait aux critères de Brighton relatifs à la certitude liée au diagnostic; la causalité n'a donc pas pu être évaluée. Un cas de myélite transverse est apparu cinq jours après l'administration d'un vaccin $R R O$, mais une infection virale concomitante en était probablement la cause. On a en outre signalé un cas de névrite optique, apparue sept jours après l'administration de la deuxième dose d'un vaccin contre le VPH. Aucune autre explication n'était apparente et le vaccin a pu être en cause. Enfin, on a signalé un seul cas d'encéphalomyélite, apparue dans les 24 heures suivant l'administration de vaccins dcaT-VPI-Hib et Pneu-C. Le 
moment de l'apparition de l'encéphalomyélite ne correspondait pas à la possibilité qu'elle puisse avoir été causée par un vaccin; on a présumé qu'une infection à entérovirus en était la cause. Les autres cas de troubles neurologiques graves sont apparus à la suite de l'administration de divers vaccins, dont aucun n'a été jugé susceptible d'avoir causé les manifestations déclarées, qui comprenaient : deux méningites à liquide clair, une paralysie de Bell, une autre paralysie d'une durée supérieure à un jour, une ataxie cérébelleuse, un AVC, deux cas de confusion temporaire, et un de trouble de conversion.

Vingt EIG ont été déclarés en rapport à des réactions au point d'injection ou près de celui-ci, notamment : 15 cas de cellulite, trois cas d'œdème sévère d'un membre, un cas d'abcès et un cas ne pouvant pas être placé dans une sous-classe. Dans tous les cas, la raison pour laquelle le cas a été jugé grave a été l'hospitalisation, principalement administration d'un antibiotique par voie intraveineuse. Les vaccins en cause incluaient : le vaccin polysaccharidique contre le pneumocoque (14 déclarations, dont sept sans autre vaccin), le vaccin antigrippal inactivé (neuf déclarations, dont trois sans autre vaccin) et deux cas associés au vaccin dcaT-VPI-Hib (un sans autre vaccin). Les symptômes sont apparus dans les 24 heures après l'immunisation dans 16 des cas, et deux jours après dans quatre des cas.

Parmi 10 manifestations allergiques graves, on comptait six déclarations de réaction anaphylactique (une de niveau 1, deux de niveau 2 et trois de niveau 4 en vertu des critères de Brighton), une de syndrome oculorespiratoire et trois de réaction allergique autre. L'apparition est survenue dans les 30 minutes dans six cas, dans un délai de 1 à < 24 heures dans deux cas, après 17 jours dans un cas et dans un délai inconnu pour un autre cas. Les vaccins associés aux réactions anaphylactiques comprenaient deux vaccins HB administrés seuls, deux vaccins antigrippaux inactivés administrés seuls et deux vaccins dcaT-VPI-Hib, un administré seul et l'autre administré avec des vaccins Men-C-C et Pneu-C. La seule manifestation inhabituelle était un cas diagnostiqué comme étant une hypersensibilité de type IV accompagnée d'une hépatite secondaire, apparue chez un adulte d'âge moyen, 17 jours après l'administration d'une deuxième dose d'un vaccin Chol-Ecol-O et 39 jours après l'administration de vaccins HAHB et Typh-I/O. La causalité n'a pas pu être évaluée de façon approfondie, en raison d'une insuffisance de renseignements, mais la personne s'est complètement rétablie.

Parmi les autres manifestations précisées dans le formulaire du SCSESSI, les déclarations d'EIG comprenaient 10 cas d'épisode hypotonique-hyporéactif, neuf de thrombocytopénie et deux d'intussusception. Aucun de ces cas n'était inattendu, étant donné les relations connues, mais plusieurs étaient explicables par d'autres raisons.

Les autres déclarations d'EIG se classaient, selon l'examen des dossiers médicaux, parmi les événements d'ordre général (52 cas), divers (20 cas) ou liés à l'anxiété due à la vaccination (deux cas), dont les manifestations touchaient une vaste gamme de diagnostics, de vaccins et de groupes d'âge particuliers. On n'a observé aucune concentration notable de cas de tout type de manifestations ou de groupe de vaccins, ni aucun fait soulevant une préoccupation.

\section{Discussion}

Le présent rapport est le premier rapport annuel sur la sécurité des vaccins administrés au Canada au cours d'une année civile présenté conformément à un modèle uniforme. L'interprétation des données du SCSESSI doit tenir compte de plusieurs mises en garde. La surveillance ne permet pas de consigner tous les événements et il n'existe par de dénominateurs fiables, relativement aux vaccins administrés; l'incidence des ESSI ne doit donc pas être calculée ni extrapolée à partir de ces données. L'exhaustivité des déclarations et leur conformité, par rapport aux définitions nationales de cas, varient. Les taux de déclaration dépendent de nombreux facteurs, notamment l'âge, le territoire de compétence, la nouveauté d'un programme de vaccination donné et le degré de controverse, au sein du public, concernant les préoccupations, réelles ou perçues, en matière d'innocuité. Alors que le présent rapport résume notre expérience des huit dernières années, les renseignements fournis au 
SCSESSI sont régulièrement examinés, au cours de l'année, en vue de déceler toute augmentation inhabituelle ou imprévue des EIG, et d'y réagir.

Le principal objectif de la surveillance après commercialisation est de détecter toute préoccupation nouvelle ou inhabituelle concernant l'innocuité pouvant signaler des liens auparavant inconnus entre un vaccin donné et un événement, ou des changements des profils prévus d'innocuité, en ce qui a trait à la fréquence ou à la gravité de certains effets indésirables. Ainsi, bien que la déclaration de liens présumés soit encouragée, il ne faut pas oublier qu'une déclaration n'est pas la preuve qu'un ou des vaccins causent un effet donné. De par leur nom même, les ESSI représentent une relation temporelle d'un effet qui suit une immunisation. La cause de cet effet peut être attribuable à une propriété intrinsèque du vaccin, à un problème de qualité de fabrication, à une erreur lors d'une étape du processus d'immunisation, à une anxiété associée à l'immunisation ou à une manifestation qui coïncide avec la vaccination, mais qui n'a aucun lien avec elle (22). II est rarement possible de déterminer une cause précise en fonction de ce qui est déclaré dans un système national comme le SCSESSI. II faut toujours effectuer une enquête plus approfondie à propos d'un signal détecté afin d'en déterminer la cause, tant au niveau de la personne que de la population. Néanmoins, les données présentées dans le présent rapport fournissent un profil au fil du temps qui peut servir à tirer des conclusions générales, en ce qui a trait au SCSESSI en tant que système de surveillance et à l'innocuité des vaccins administrés au Canada.

D'une part, le Canada dispose d'un solide système de surveillance des vaccins fondé sur des décennies de collaboration synergique avec les autorités sanitaires publiques régionales et fédérales, ainsi qu'avec des partenaires clés, décrits plus en détail ailleurs dans le présent supplément. Au Canada, le taux de déclaration total annuel d'ESSI de 10,1 pour 100000 habitants est élevé, si on le compare à la plupart des autres pays dont le calendrier d'immunisation est similaire, notamment les États-Unis (taux de 4,4 pour les vaccins administrés de 1991 à 2001) (13), l'Europe (taux de 2005 inférieur à 5 en France, en Allemagne, en Grande-Bretagne, en Italie et en Espagne, de 6 ou 7 aux Pays-Bas et en Norvège, de 9,8 en Suède et de 18,5 en Finlande) (43), et semble comparable à celui de l'Australie (10,4 en 2011), mais est probablement supérieur (14). Le Canada et la plupart des autres pays utilisent les déclarations de cas individuels comme le numérateur des taux représentatifs de la population, alors que l'Australie se sert des effets indésirables individuels, qui excèdent souvent le nombre de un par cas déclaré. En fonction de programmes d'immunisation similaires, les taux de déclaration d'ESSI supérieurs du Canada reflètent le haut niveau de vigilance des autorités sanitaires publiques qui déclarent les ESSI, ainsi que du volet de surveillance active du réseau IMPACT.

D'autre part, le profil des vaccins administrés au Canada est très bon. La vaste majorité des ESSI signalés sont de faible gravité et se résorbent complètement. La seule concentration notable de cas d'effets graves associés à des vaccins administrés en 2012 concernait des convulsions fébriles au cours de la deuxième année de vie, probablement attribuables à des vaccins contenant le virus de la rougeole, et dont le taux le plus élevé était lié au vaccin RORV. Bien qu'il s'agisse d'un effet attendu, il effraie les personnes concernées. Il est essentiel que des renseignements réalistes à propos du rapport entre les risques et les avantages, ainsi que des mesures à prendre le cas échéant, soient communiqués aux parents et aux personnes soignantes.

Enfin, la classification de l'examen du dossier médical, entreprise à l'échelle nationale en 2011 par la Section de la sécurité des vaccins de l'Agence de la santé publique du Canada (ASPC), offre un cadre additionnel en vue de soumettre les données relatives aux ESSI et qui sera, espère-t-on, plus utile aux intervenants cliniques, publics et des autorités sanitaires que les rapports sommaires précédents fondés sur des cadres réglementaires de codage comme MedDRA. Les deux cadres sont utiles et c'est pourquoi ils sont inclus dans le présent rapport. Les données de la Classe par système et organe procurent un moyen de comparaison entre le présent rapport et ceux publiés par les organismes nationaux de réglementation et les détenteurs d'une autorisation de mise en marché. La classification de l'examen du dossier médical est étroitement liée avec le formulaire de déclaration du SCSESSI et sert également à la rédaction des résumés des ESSI signalés que l'ASPC publiée trimestriellement en ligne (44). La classification de l'examen du dossier médical constitue aussi un outil additionnel important en 
vue de permettre aux spécialistes d'effectuer un examen opportun de tous les EIG signalés à l'ASPC et de faciliter les activités d'assurance de la qualité.

\section{Remerciements}

Le présent rapport n'aurait pu être réalisé sans la contribution de tous les participants au SCSESSI, notamment les autorités fédérales, provinciales et territoriales d'immunisation, particulièrement celles qui font partie du Groupe de travail sur la vaccinovigilance, le réseau IMPACT et tout le personnel de la Section de la sécurité des vaccins de l'ASPC. Nous aimerions également remercier toutes les personnes ayant pris le temps de déclarer des ESSI pour leur contribution à la sécurité des vaccins au Canada.

\section{Conflit d'intérêts}

Aucun

\section{Financement}

Ce travail a été entièrement financé par l'Agence de la santé publique du Canada.

\section{Références}

(1) Childhood Immunization Division, Bureau of Communicable Disease Epidemiology, Laboratory Centre for Disease Control, Health and Welfare Canada. Synopsis of a workshop on post-marketing surveillance of vaccine-associated adverse events. Canada Diseases Weekly Report. 1991; 17-19:97-8.

(2) Koch J, Leet C, McCarthy R, Carter A, Cuff W, Disease Surveillance Division, Bureau of Communicable Disease Epidemiology, Laboratory Centre for Disease Control. Adverse events temporally associated with immunizing agents-1987 report. CMAJ. 1989; 141:933-7.

(3) Duclos P, McCarthy R, Koch J, Carter A. Bureau of Communicable Disease Epidemiology, Laboratory Centre for Disease Control. Adverse events temporally associated with immunizing agents-1988 report. Canada Diseases Weekly Report. 1990; 16-32:157-64.

(4) Duclos P, Koch J, Hardy M, Carter A, McCarthy R. Bureau of Communicable Disease Epidemiology, Laboratory Centre for Disease Control. Adverse events temporally associated with immunizing agents—1989 report. Canada Diseases Weekly Report. 1991; 17-29:147-56.

(5) Duclos P, Pless R, Koch J, Hardy M. Adverse events temporally associated with immunizing agents. Can Fam Physician. 1993; 39:1907-13.

(6) Childhood Immunization Division, Bureau of Communicable Disease Epidemiology, Laboratory Centre for Disease Control. Adverse events temporally associated with immunizing agents-1991 report. CCDR. 1993; 19-20:168-79.

(7) Bentsi-Enchill A, Hardy M, Koch J, Duclos P. Effets secondaires reliés dans le temps à des vaccins - rapport de 1992. RMTC 1995; 21-13:F1-F9.

(8) Rapport sur l'immunisation au Canada, 1996. Chapitre 9. Surveillance des incidents indésirables associés au moment de l'administration du vaccin. RMTC. 1997; 23 Suppl 4:S24-7.

(9) Canadian National Report on Immunization, 1997. Paediatrics and Child Health. 1998; 3 Suppl B:25B-8B.

(10) 1998 National Report (interim) on Immunization Vaccine Safety Issues and Surveillance, Paediatrics and Child Health. 1999; 4 Suppl C:26C-9C.

(11) Agence de la santé publique du Canada. Rapport national sur l'immunisation au Canada, 2006. Relevé des maladies transmissibles au Canada, vol. 32(suppl. 3):S29-36, 2006.

(12) Ontario Agency for Health Protection and Promotion (Public Health Ontario). Annual Report on Vaccine Safety in Ontario, 2012. Technical Report. Toronto, ON: Queen's Printer for Ontario; 2014. http://www.publichealthontario.ca/en/eRepository/Annual_Report_Vaccine_Safety_Ontario_2012.pdf

(13) Zhou W, Pool V, Iskander JK, et al. Surveillance for safety after immunization: Vaccine adverse event reporting system (VAERS)—United States, 1991-2001. MMWR. 2003; 52:1-10.

(14) Mahajan D, Cook J, Dey A, Macartney K, Menzies RI. Annual Report: Surveillance of adverse events following immunization in Australia, 2011. Commun Dis Intell. 2012; 36:E315-32.

(15) Agence de la santé publique du Canada. Système canadien de surveillance des effets secondaires suivant l'immunisation [mis à jour le 7 juillet 2014]. 
http://www.phac-aspc.gc.ca/im/vs-sv/index-fra.php

(16) Agence de la santé publique du Canada. Déclaration de manifestations cliniques inhabituelles ( $\mathrm{MCl})$ à la suite d'une immunisation. http://www.phac-aspc.gc.ca/im/pdf/raefi-dmcisi-fra.pdf

(17) Agence de la santé publique du Canada. F/P/T Renseignements des personnes contact pour les questions liées aux effets secondaires suivant l'immunisation. [consulté le 30 juillet 2012]. http://www.phac-aspc.gc.ca/im/ci-rp-fra.php

(18) Société canadienne de pédiatrie. Programme canadien de surveillance active de l'immunisation. La Surveillance [mis à jour le 13 août 2014]. http://www.cps.ca/fr/impact

(19) Morris R, Halperin S, Dery P, Mills E, Lebel M, MacDonald N, Gold R, Law B, Jadavji T, Scheifele D, Marchessault V, Duclos P. IMPACT monitoring network: A better mousetrap. Can J Infect Dis. 1993; 4:194-5.

(20) Scheifele DW, Halperin SA, and members of the Health Canada/CPS Immunization Monitoring Program, Active (IMPACT). A Model of Active Surveillance of Vaccine Safety. Seminars in Pediatric Infectious Diseases. 2003 Jul; 14(3):213-9.

(21) Agence de la santé publique du Canada. Guide canadien d'immunisation, Partie 2, Innocuité des vaccins [mis à jour le 4 décembre 2013]. http://www.phac-aspc.gc.ca/publicat/cig-gci/p02-fra.php

(22) Council for International Organizations of Medical Sciences (CIOMS) and World Health Organization (WHO). Definition and Application of Terms for Vaccine Pharmacovigilance. Report of CIOMS/WHO Working Group on Vaccine Pharmacovigilance. Geneva: CIOMS and WHO; 2012. http://whqlibdoc.who.int/publications/2012/9789290360834_eng.pdf

(23) International Conference on Harmonisation (ICH) of Technical Requirements for Registration of Pharmaceuticals for Human Use. ICH Harmonised Tripartite Guideline. Clinical Safety Data Management: Definitions and Standards for Expedited Reporting E2A. Current Step 4 version. 1994 Oct 27. http://www.ich.org/fileadmin/Public_Web_Site/ICH_Products/Guidelines/Efficacy/E2A/Step4/E2A_Guideline.pdf

(24) Marcy SM, Kohl KS, Dagan R, et al. Fever as an adverse event following immunization: Case definition and guidelines of data collection, analysis and presentation. Vaccine. 2004; 22:551-6.

(25) Halperin S, Kohl KS, Gidudu J, et al. Cellulitis at injection site: Case definition and guidelines for collection, analysis, and presentation of immunization safety data. Vaccine. 2007; 25:5803-20.

(26) Kohl KS, Ball L, Gidudu J, et al. Abscess at injection site: Case definition and guidelines for collection, analysis, and presentation of immunization safety data. Vaccine. 2007; 25:5821-38.

(27) Ruggeberg JU, Gold MS, Bayas JM, et al. Anaphylaxis: Case definition and guidelines for data collection, analysis and presentation of immunization safety data. Vaccine. 2007; 25:5675-84.

(28) Sejvar JJ, Kohl KS, Bilynsky R, et al. Encephalitis, myelitis, and acute disseminated encephalomyelitis (ADEM): Case definition and guidelines for collection, analysis, and presentation of immunization safety data. Vaccine. 2007; 25:5771-92.

(29) Tapianen T, Prevots R, Izurieta HS, et al. Aseptic meningitis: Case definition and guidelines for collection, analysis, and presentation of immunization safety data. Vaccine. 2007; 25:5793-802.

(30) Sejvar JJ, Kohl KS, Gidudu J, et al. Guillain-Barré syndrome and Fisher syndrome: Case definition and guidelines for collection, analysis, and presentation of immunization safety data. Vaccine. 2011; 29:599-612.

(31) Bonhoeffer J, Menkes J, Gold MS, et al. Generalized convulsive seizure as an adverse event following immunization: Case definition and guidelines for data collection, analysis, and presentation. Vaccine. 2004; 22:557-62.

(32) Buettcher M, Heininger U, Braun M, et al. Hypotonic-hyporesponsive episode(HHE) as an adverse event following immunization in early childhood: Case definition and guidelines for collection, analysis, and presentation of immunization safety data. Vaccine. 2007; 25:5875-81.

(33) Bines JE, Kohl KS, Forster J, et al. Acute intussusception in infants and children as an adverse event following immunization: Case definition and guidelines of data collection, analysis, and presentation. Vaccine. 2004; 22:569-74.

(34) Bonhoeffer J, Vermeer P, Halperin S, et al. Persistent crying in infants and children as an adverse event following immunization: Case definition and guidelines for data collection, analysis, and presentation. Vaccine. 2004; 22:586-91.

(35) Beigel J, Kohl KS, Khuri-Bulos N, et al. Rash including mucosal involvement: Case definition and guidelines for collection, analysis, and presentation of immunization safety data. Vaccine. 2007; 25:5697-706.

(36) Wise RP, Bonhoeffer J, Beeler J, et al. Thrombocytopenia: Case definition and guidelines for collection, analysis, and presentation of immunization safety data. Vaccine. 2007; 25:5717-24. 
(37) Jorch G, Tapiainen T, Bonhoeffer J, et al. Unexplained sudden death, including sudden infant death syndrome (SIDS), in the first and second years of life: Case definition and guidelines for collection, analysis and presentation of immunization safety data. Vaccine. 2007; 25:5707-16.

(38) Agence de la santé publique du Canada (ASPC) et Groupe de travail sur la vaccinovigilance. Déclaration de manifestations cliniques inhabituelles $(\mathrm{MCl})$ à la suite d'une immunisation au Canada : Guide de l'utilisateur pour remplir et soumettre les rapports de déclaration de MCI. Ottawa : ASPC; 2011. p. 18-25. http://www.phac-aspc.gc.ca/im/aefi-essi_guide/index-fra.phpf

(39) Woo EJ, Burwen DR, Gatumu SNM, et al. Extensive limb swelling after immunization: Reports to the Vaccine Adverse Event Reporting System. Clinical Infectious Diseases. 2003; 37:31-8.

(40) van der Maas NA, Bondt PE, de Melker H, Kemmeren JM. Acute cerebellar ataxia in the Netherlands: A study on the association with vaccinations and VZV infection. Vaccine. 2009; 27:1970-3.

(41) Statistique Canada. Estimations démographiques annuelles : Canada, provinces et territoires [mis à jour le 26 septembre 2014]. http://www5.statcan.gc.ca/olc-cel/olc.action?Objld=91-215-X\&ObjType=2\&lang=fr\&limit=0

(42) MacDonald SE, Dover DC, Simmonds KA, Svenson LW. Risk of febrile seizures after first dose of measles-mumpsrubella-varicella vaccine: A population-based cohort study. CMAJ. 2014; 186:824-9.

(43) Zanoni G, Berra P, Ilaria L, et al. Vaccine adverse event monitoring systems across the european Union countries: Time for unifying efforts. Vaccine. 2009; 27:3376-84.

(44) Agence de la santé publique du Canada. Effets secondaires suivant l'immunisation (ESSI) : Rapport trimestriel pour 2014 - T2. http://www.phac-aspc.gc.ca/im/vs-sv/aefi-essi-2014-q2-fra.php 